a dispersion of $5 \mathrm{I} \mathrm{A} / \mathrm{mm}$ at $H \gamma$ were secured between January 8 and October 19 , this year. The following orbital elements were deduced from twenty-seven plates on which the velocities of both components were measured.

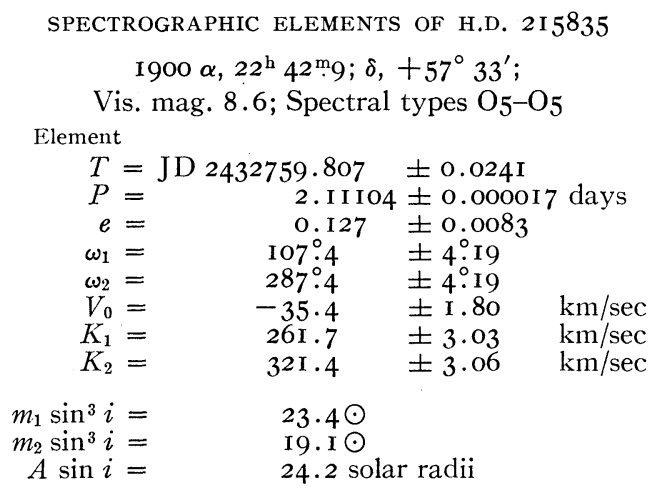

The radial velocity of the strong interstellar $\mathrm{K}$-line measured on every plate is $-26.8 \pm 0.30$ $\mathrm{km} / \mathrm{sec}$. The intensity of the K-line was measured on five plates, from which a distance of 2 IOO parsecs was derived. This agrees well with Trumpler's adopted value of 1840 parsecs for the cluster.

The components have the earliest spectral types of all spectrographic binaries. The stars have similar spectra, being normal absorption $\mathrm{O}_{5}$ stars. The difference in magnitude, $\Delta m=0.28$, was spectrophotometrically determined from the intensities of $\lambda 4542$ and $H \gamma$ on six plates.

The following physical characteristics were deduced upon the assumption that the stars are normal O-type stars, conforming to Eddington's mass-luminosity relation.

The orbital inclination is estimated to be $62^{\circ}$ and an eclipse of $\mathrm{o} . \mathrm{I}$ mag. at primary minimum is predicted.

No observations of the tenth magnitude class $B$ stars in this cluster have been obtained at Victoria, B. C. It is to be observed, however, that the systemic velocity of HD 215835 is 4.6 $\mathrm{km} / \mathrm{sec}$, more positive than Trumpler's provisional value, so that the present investigation does not support his relativity red-shift hypothesis. The estimated maximum mass of $62 \odot$ while quite large is quite normal for this interesting O-type system. The star is being observed by Dr. Kron at the Lick Observatory in order to determine an accurate light curve.

Dominion Astrophysical Observatory, Victoria, B. C.

\section{Sen, Hari K. The radiative equilibrium of a spherical planetary nebula.}

The effect of curvature of the layers of a spherical planetary nebula on the fields of ultraviolet as well as Lyman- $\alpha$ radiation has been investigated by the spherical harmonic method. Allowing for the dilution of the radiation in the extended nebular shell, the following effects have been found, which may be attributed to the curvature of the nebular shell:

(I) The ultra-violet flux monotonically increases from the inner to the outer boundary of the nebula (the plane case has a maximum in the range), and is greater than that of the plane nebula by a factor of 2 to 3 .

(2) The $\mathrm{L}_{\alpha}$ flux for the static nebula increases as in the plane nebula monotonically from the inner to the outer boundary of the nebula, but is less than that of the plane nebula by a factor of $2 / 3$.

(3) If the nebula be supposed to expand with a uniform velocity large compared with the mean velocity of molecular motion, the $\mathrm{L}_{\alpha}$ fluxes for the moving plane and spherical nebulae are of the same order of magnitude towards the outer parts; the flux decreases much more rapidly towards the interior in the plane nebula, so that the inner parts which suffer inward radiative accelerations are much more extensive in the plane than in the spherical nebula.

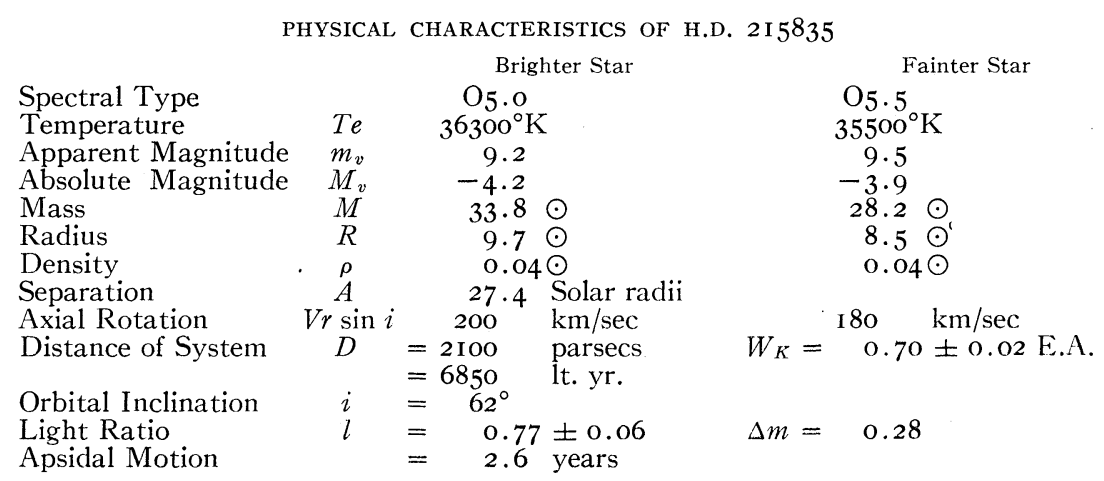


The present analysis for the spherical nebula reduces in the limit to Chandrasekhar's corresponding analysis for the plane nebula. ${ }^{1}$ Allowing for the dilution factor, the difference between the fluxes in $\mathrm{L}_{\alpha}$ for the moving and the static nebula is found to be a constant, as in the plane case. The two constants are, however, different, and are equal only in the limiting case when the sphere reduces to a plane.

I. Zs. Astroph. 9, 266, 1935.

Harvard College Observatory, Cambridge, Mass.

\section{Struve, Otto. The spectrum of the cluster-type variable CY Aquarii.}

The cluster-type variable CY Aquarii has the shortest period among the I0,9I2 variables listed in the recent catalogue by Kukarkin and Parenago. It is only 88 minutes or 0.061038476 day. Gossner and Ashbrook ${ }^{1}$ have found that the period is probably variable but the above value determined by Wesselink is sufficiently accurate for our purpose. The brightness changes between I0.5 and II.4 on the photographic scale. Hence, it was necessary to use a spectrograph of relatively small dispersion in order to make the exposure times short enough. With the Cassegrain spectrograph of the McDonald Observatory equipped with two quartz prisms and a Schmidt camera of $\mathrm{I} 80 \mathrm{~mm}$ focal length the linear dispersion is $100 \mathrm{~A} / \mathrm{mm}$ at $\lambda 3933$. With this dispersion the exposure times for well broadened spectrograms range between about to minutes at maximum light and 24 minutes at minimum light. The hydrogen lines are strong at maximum and the spectrum does not look abnormal but near minimum light the hydrogen lines are weak as compared to the line of $C a$ II 3933 and the metallic lines, though weakly present, are not as strong as one would expect. Radial velocities have been determined from II members of the Balmer series and from the line $C a$ II 3933 . The results are shown in Figure I. Phase zero corresponds

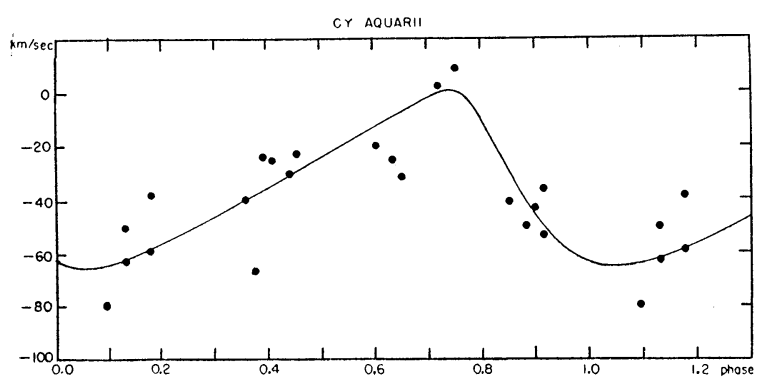

to maximum light. The radial velocity is then at its lowest value, namely about $-64 \mathrm{~km} / \mathrm{sec}$. The maximum radial velocity of about $\mathrm{o} \mathrm{km} / \mathrm{sec}$ is reached at about phase 0.75 which corresponds approximately to the minimum of the light curve. The total range is of the order of $64 \mathrm{~km} / \mathrm{sec}$ which is very nearly the same as that observed in RR Lyrae and XZ Cygni. There is thus no conspicuous relation between the amplitude of the velocity curve and the period. It should also be pointed out that among the cluster-type variables there is no pronounced relation between median spectral type and period as there is among the classical Cepheids. The average spectral type of CY Aquarii is only very slightly earlier than that of RR Lyrae and XZ Cygni and is not as early as was believed by Wachmann who had tentatively announced a variation from B8 to A3. The average radial velocity of the variable is about $-32 \mathrm{~km} / \mathrm{sec}$.

I. A. J. 52, 55, 1946.

Yerkes Observatory, Williams Bay, Wis. and McDonald Observatory Fort Davis, Tex.

\section{Thomas, R. N. On emission lines at high kinetic temperature.}

If one admits the existence of a general outer stellar atmosphere whose kinetic temperature exceeds the radiation temperature of the star, some other mode of energy transfer than radiation is implied. There exists thus the possibility of increasing the emission in certain spectral regions without requiring a diminution in others. The conditions for a net chromospheric emission under conditions of high kinetic temperature and non-thermodynamic equilibrium are presented, with results in general considerably different from the earlier conclusions of Menzel. The most pressing refinement needed for the general case over the type calculations already made for the solar chromosphere, where Lyman emission and Balmer absorption were found, lies in the consideration of collisional excitation from the $n=2$ level. The inclusion of such a term may force the earlier Balmer members into emission under conditions of sufficiently large electron density and kinetic temperature. It is suggested that the phenomenon may find application in the transitory bright line spectrum observed by Struve in RR Lyrae.

Institute of Advanced Study, Princeton University, Princeton, N. J. 